\title{
Management of chronic pain in the elderly: focus on transdermal buprenorphine
}

\author{
Nalini Vadivelu \\ Roberta L Hines \\ Department of Anesthesiology, Yale \\ University School of Medicine, New \\ Haven, USA
}

\begin{abstract}
Chronic pain in the elderly is a significant problem. Pharmacokinetic and metabolic changes associated with increased age makes the elderly vulnerable to side effects and overdosing associated with analgesic agents. Therefore the management of chronic cancer pain and chronic nonmalignant pain in this growing population is an ongoing challenge. New routes of administration have opened up new treatment options to meet this challenge. The transdermal buprenorphine matrix allows for slow release of buprenorphine and damage does not produce dose dumping. In addition the long-acting analgesic property and relative safety profile makes it a suitable choice for the treatment of chronic pain in the elderly. Its safe use in the presence of renal failure makes it an attractive choice for older individuals. Recent scientific studies have shown no evidence of a ceiling dose of analgesia in man but only a ceiling effect for respiratory depression, increasing its safety profile. It appears that transdermal buprenorphine can be used in clinical practice safely and efficaciously for treating chronic pain in the elderly.
\end{abstract}

Keywords: transdermal buprenorphine, chronic pain, elderly

\section{Introduction}

Many chronic pain patients are elderly (ie, $>65$ years of age) (Sittl 2006) and a significant number of these experience chronic pain which can affect their normal functioning and quality of life (Gagliese and Melzack 1997). Difficulty in assessing the quality and intensity of pain due to cognitive changes and the presence of multiple medical problems associated with aging contributes to undertreatment of pain in this population. However, pharmacokinetic and metabolic changes associated with increased age make these patients more vulnerable to the potential side effects and overdosing with analgesic agents. Therefore, management of chronic pain due to cancer or persistent neuropathic pain (McQuay 2002; Foley 2003) is a challenge, especially in the growing population of elderly patients.

Chronic pain treatment in the elderly is multidimensional and includes noninvasive as well as invasive therapies. Invasive therapies include pharmacological therapies with nonopioid, opioid, and adjuvant medications. New routes of administration of drugs have opened up new treatment options for the treatment of chronic pain in the elderly (Grond et al 2000). In this context buprenorphine, a semi-synthetic, lipophilic, unique opioid analgesic, has broad clinical applications (Vadivelu and Hines 2007). Buprenorphine is being used sublingually (Book et al 2007) and intrathecally (Shah et al 2003) for the treatment of acute pain and transdermally for the treatment of chronic pain (Graziotti and Goucke 1997; Simpson 2002; Budd 2003; Likar et al 2003). Its long-acting analgesic property and relative safety administered in this form make it useful for the treatment of chronic pain in the elderly. This review focuses on the use of transdermal buprenorphine for the treatment of chronic pain in the elderly. 


\section{Chronic pain management in the elderly}

Chronic pain increases with age (Crook et al 1984) due to increases in the incidence of diseases such as arthritis, cancer, diabetes mellitus, and cardiovascular and neurological diseases (Priano et al 2006). Detection and management of chronic pain in the elderly is often inadequate and continues to pose a challenge for practitioners (Woo et al 1994). The detection of chronic pain in the elderly can be done with a pain scoring pain system which assesses the severity of the patient's pain subjectively on a score of from 1 to 10 . The detection of pain in the elderly may be complicated by the presence depression and dementia. Dementia and confusion make the assessment of pain sometimes unreliable with resultant difficulty in applying the conservative and interventional therapies in these patients.

Elderly patients with depression report more pain than those who are not depressed (Casten et al 1995), and complaints of pain may be the first sign of dementia (Kisely et al 1992). Chronic pain in the elderly can be either nociceptive or neuropathic. The conservative therapies for these states include adequate nutrition, cognitive and behavioral therapies, rehabilitational pain medicine, biofeedback, relaxation, and alternative medicine techniques such as acupuncture and acupressure. Low level activity programs also can benefit the elderly physically (Helme et al 1989).

Nonsteroidal anti-inflammatory drugs are commonly used to treat nociceptive pain and inhibit prostaglandin production. Malignant pain is often treated in the elderly with opioids which can be used also for treatment of neuropathic nonmalignant pain. Due to high fat to protein ratios, opioids in the elderly should be started at doses $25 \%-50 \%$ lower than those used in young adults, with rescue doses limited to $5 \%$ of the total daily dose available every 4 hours (Abrahm 2000). Commonly used opioids in the elderly are oxycodone, morphine, hydromorphone, and fentanyl patch. Oxycodone is a preferred drug in the elderly since it has a short half-life and no toxic metabolites, and is available in both the shortand long-acting forms. Morphine can be used with caution, paying attention to renal insufficiency and sedation.

Common adjuvant medications for the treatment of nonmalignant neuropathic pain in the elderly include a variety of medications such as nonopioid analgesics, opioid analgesics, adjuvant medications including antidepressants, anticonvulsants, tramadol, and capsaicin. The invasive techniques include neuromodulatory or surgical interventions such as nerve blocking, spinal cord stimulation and intraspinal, implantable drug delivery, and neurodestructive interventions. It is recommended that the least invasive and simple interventions be tried first in the elderly before use of more invasive interventions.

Chronic pain control in the elderly by all these therapeutic measures is still inadequate. The recent development of transdermal buprenorphine has opened up new treatment options for treatment of chronic pain in this growing patient group. It has unique pharmacodynamics which could make it a useful analgesic in the presence of age-related cognitive, metabolic, and pharmacokinetic changes so often seen in the elderly. The pharmacokinetics of buprenorphine, especially its slow association and disassociation rate at the receptor sites, make it useful for use in the elderly (Yassen et al 2005).

\section{Pharmacodynamics of buprenorphine}

Originally, buprenorphine was thought to be 25-50 times more potent by weight than morphine in an equivalent dose (Jasinski et al 1978), but it is now thought that buprenorphine is $75-100$ times more potent than morphine (Sittl et al 2006). Buprenorphine is a centrally acting partial mu agonist and a kappa and delta opioid receptor antagonist (Negus et al 2002). Buprenorphine has a high affinity for the mu receptor and a lower intrinsic activity than a full agonist mu opioid receptor agonist (Cleeland et al 1994). It appears that the mu agonist effect is most important for producing its analgesic results. Buprenorphine can produce near maximal mu receptor occupation, thereby decreasing mu opioid receptor availability, and replace agonist effects and alleviate withdrawal symptoms (Greenwald et al 2003). Buprenorphine produces stabilization of mu receptors in the submembrane level and increases the expression of mu receptors on membranes (Evans and Easthope 2003). By affecting the mu receptor, reserve buprenrophine can enable the switch from another opioid to buprenorphine. The mu agonist effect of buprenorphine is in the form of a bell-shaped curve. In animal models, the bell-shaped analgesic dose response curve peaks at $1 \mathrm{mg} / \mathrm{kg}$, which is below the highest dose of $32 \mathrm{mg} / \mathrm{kg}$ reported in humans.

Buprenorphine interacts with orphanin $\mathrm{FQ}$ /nociceptin receptor ORL-1 in the spinal cord and the brain stem. Buprenorphine activates ORL1 in the spinal cord, which appears to be analgesic. OR-1 activation in the brain stem blocks opioid analgesic responses and contributes to the partial agonist property of buprenorphine (Bloms-Funke et al 2000). Buprenorphine also possesses an antihyperalgesic effect relative to clinically used mu agonists; its antihyperalgesic effects exceed its analgesic effects (Simonnet and Rivat 2003; 
Koppert et al 2005; Simonnet 2005). It is thought that the antihyperalgesic effect of buprenorphine may be related to kappa opioid receptor blockade.

Buprenorphine is associated with a long duration of action, 6-8 hours (Johnson et al 2005), which has been attributed to the slow dissociation of buprenorphine from the mu receptor (Jasinski et al 1978). The transdermal buprenorphine formulation has a slow onset (12-24 hours) and a long duration of action (3 days) (Sorge and Sittl 2004). Increased sensitivity to the depressant actions of opioids in the elderly is well established. Fentanyl as a transdermal preparation is not recommended for used in opioid-naïve patients because of the incidence of respiratory depression (Thompson et al 1998).

Clinically relevant doses of up to $10 \mathrm{mg}$ of buprenorphine have shown full dose-dependent effects on analgesia with no respiratory depression (Dahan et al 2005). Dahan et al (2006) have shown a ceiling to respiratory depression but not to analgesia over a dose range from 0.05 to $0.6 \mathrm{mg}$ buprenorphine in humans This safety profile could give transdermal buprenorphine a preferred role over transdermal fentanyl for treatment of chronic pain in the elderly. In humans, there is also a less marked effect of buprenorphine binding to mu opioid receptors on gastrointestinal transit times, and indeed constipation seen in the clinic is remarkably low (Griessinger et al 2005).

There was initial concern that the partial agonism of buprenorphine would lead to limited analgesia and would prevent other opioids from effectively binding. It has since been shown that buprenorphine is a strong analgesic, with no ceiling effect for analgesia (Jasinski et al 1978; Budd and Collett 2003). It does not produce persistent binding of the mu receptor, which would prevent the action of other opioids in acute and chronic painful conditions (Walsh et al 1995); as a result other opioids can be used for breakthrough pain in the presence of buprenorphine (Budd and Collett 2003).

\section{Decreased tolerance}

In humans exposed to opioids, but not physically dependent on them, a study of volunteers receiving sublingual buprenorphine in ascending doses of 1 to $32 \mathrm{mg}$ demonstrated no ceiling effect for its analgesia effects (Walsh et al 1994). In physically dependent individuals, acute cessation of buprenorphine may lead to withdrawal symptoms that appear to be milder than those seen with morphine (Heel et al 1979). Zaki et al (2000) studied equal concentrations (10 $\mathrm{MM} / \mathrm{L})$ of morphine, fentanyl, and buprenorphine, and showed that buprenorphine did not induce the loss of opioid receptors from the cell surface, as did morphine and fentanyl. Buprenorphine was unable to trigger mu receptor internalization because it has a low efficacy for activating G proteins. Sittl et al (2005), who studied the use of transdermal fentanyl and transdermal buprenorphine in humans, found a significant increase in the mean daily dose of fentanyl over buprenorphine in this time period. The long analgesic action of buprenorphine as well as its decreased tolerance because of loss of opioid receptors from the cell surface make it suitable the treatment of chronic pain in the elderly.

\section{Metabolism and elimination}

The metabolism of buprenorphine is mediated by cytochrome P450 in the liver (Heel et al 1979). Buprenorphine is oxidized to $\mathrm{N}$ dealkyl buprenorphine, also called norbuprenorphine by CYP3A4. Most opioids with the exception of buprenorphine and morphine undergo oxidation, and buprenorphine and morphine undergo glucuronidation. Both buprenorphine and norbuprinorphine undergo rapid glucuronidation. The binding to glucuronic acid leads to a low bioavailability of 5\% with oral buprenorphine. Norbuprenorphine is 10 times more potent than buprenorphine in causing respiratory depression. However, this respiratory depressant effect of norbuprenorphine can be antagonized by naloxone (Gal 1989) and also by the substance beta-flunaltrexamine. It also has a weak mu agonist effect that is seen after chronic use. Most of the concentration of norbuprenorphine has been seen in the lungs rather than in the brain (Ohtani et al 1997).

Buprenorphine is metabolized to glucuronide metabolites which can be hydrolyzed in the intestine to release buprenorphine again. The released buprenorphine can be reabsorbed via the enterohepatic circulation. Excretion of buprenorphine and metabolites is $70 \%$ by feces of unchanged buprenorphine, conjugated buprenorphine, and the Phase I conjugated metabolites. The other small quantity of buprenorphinerelated substances is excreted in the urine (Budd 2003). Unconjugated buprenorphine does not appear in urine, only in stool secondary to fecal flora beta-glucuronidase (Cone et al 1984).

\section{Age-related changes in drug kinetics and dynamics relevant to opioids}

The narrowing of the therapeutic index because of physiological changes in aging can alter the pharmacokinetics and pharmacodynamics of analgesics. Important changes are seen with age which can affect opioid drug kinetics and dynamics. Hepatic blood flow decreases with age and 
age-related changes occur in cytochromes and conjugases are. These changes can narrow the therapeutic index and increase the risk of toxicity and drug-drug interactions (Davis and Srivastava 2003). Distribution of drugs to the central nervous system (CNS) are altered with alterations in receptors and transmitters. These $\mathrm{CNS}$ changes can increase risk of delirium. In addition changes in renal function can be age-related.

\section{Buprenorphine in the presence renal impairment}

Renal impairment leading to a decrease in renal excretory function is common in elderly patients due to advanced age (Balázs et al 2008) or to co-morbid conditions such as diabetes or hypertension. Buprenorphine for treatment of pain can be administered in normal doses in the elderly, because it is excreted mainly in the liver (Brewster et al 1981; Cone et al 1984). In contrast, most opioids have to be cleared by the kidney and so have to be used in smaller doses, or with decreased frequency or not used at all in the elderly. Though the levels of norbuprenorphine may be increased by continuous intravenous infusions of buprenorphine in renal failure (Summerfield et al 1985), it most likely does not produce untoward effects because of the lower potency and lower affinity of norbuprenorphine compared with buprenorphine (Hand et al 1990). Buprenorphine can be used in elderly patients with impaired renal function and chronic renal insufficiency, and in hemodialysis patients in whom its pharmacokinetics are unchanged. Filitz et al (2006) studied the disposition of buprenorphine and its metabolite norbuprenorphine in 10 patients with end-stage kidney disease treated with transdermal buprenorphine. The blood samples studied showed no increase in levels of buprenorphine and norbuprenorphine at up to $70 \mu \mathrm{g}$ /hour transdermal buprenorphine. Stable analgesic effects as seen by unchanged buprenorphine plasma levels in the presence of hemodialysis were also seen in the presence of a transdermal buprenorphine patch. Its safety for use in the presence of renal impairment is a major advantage over other opioids in the elderly.

\section{Buprenorphine in hepatic disease}

Buprenorphine at high doses increases liver enzymes due to accumulation within mitochondria. Liver function is important because buprenorphine is metabolized by CYP3A4, resulting in norbuprenorphine. Both are rapidly glucuronidated by UCT1A1 and UGT2B7, and these processes occur in the liver. Unconjugated buprenorphine is excreted in the bile (Kintz 2002; Clarot et al 2003).
Buprenorphine pharmacokinetics are stable in mild to moderate hepatic impairment (Johnson et al 2005).

\section{Side effects}

Buprenorphine can cause nausea, vomiting, sedation, euphoria, papillary constriction, delayed gastric emptying, and respiratory depression. Buprenorphine in high doses can increase liver enzymes due to accumulation within mitochondria.

Sedation should be observed with the use of buprenorphine because sedatives are known to cause pharmacodynamic interactions (Clarot et al 2003). In humans, dose-response studies on respiratory depressive response showed data characterized by a bell-shaped curve in which respiratory depression response increases with increasing dose of buprenorphine in the lower dose ranges (Walsh et al 1995). This bell-shaped effect for respiratory depression suggests a decreased likelihood of respiratory depression with buprenorphine at higher doses, suggesting increased safety of the drug. The same bell-shaped curve has not been shown for the other buprenorphine-related mu opioid agonist effects of analgesia, euphoria, sedation, and papillary constriction (Jasinski et al 1978). Nausea, vomiting, and constipation can occur with buprenorphine as with other opioids; however, it has been shown that these side effects occur significantly less with buprenorphine than with morphine (Walsh et al 1994).

\section{Comparison of side effects with other opioids}

Buprenorphine appears to have a greater margin of safety than other potent opioids such as fentanyl when used to treat chronic pain. Dahan et al (2005) compared the respiratory effects of buprenorphine and fentanyl given intravenously in healthy human volunteers. Buprenorphine-induced respiratory depression had a maximum (ceiling) effect at doses of $>0.1 \mathrm{mg} / 70 \mathrm{~kg}$ (Dahan et al 2005). In another study by Dahan et al (2006), a ceiling effect in respiratory depression, but not in analgesia, was demonstrated in a study on 20 volunteers over a weight-adjusted dose range of $0.2-0.4$ $\mathrm{mg} / 70 \mathrm{~kg}$. The peak analgesic effect of buprenorphine was increased by doubling the dose, and the timing and magnitude of respiratory depression as seen by sedation and respiratory rate remained unchanged. Of note, its analgesic effect had no plateau. Buprenorphine may thus have full agonist effect for analgesia and a partial agonist effect for respiratory depression at mu receptors, showing a differential effect on analgesia and respiration. It appears that its respiratory effects are limited, unlike morphine and fentanyl which 
have been shown to have no ceiling effect for analgesia but which can cause severe respiratory depression and apnea in high doses. No detrimental effect from chronic exposure to buprenorphine has been shown on corticosensitive immune parameters (D'Elia et al 2003). Gomez-Flores and Weber (2000) showed that buprenorphine, unlike morphine, was not associated with immunosuppression and did not activate the hypothalamic-pituatary axis. The lack of effects on the immune system is thought to be related to its partial agonist effects.

\section{Routes of administration}

Several new routes for delivery of the buprenorphine are now available and have been used to provide analgesia, including the buprenorphine/naloxone combination and transdermal buprenorphine. The parenteral forms of buprenorphine, such as subcutaneous, intramuscular, epidural, and intrathecal, have been available since 1981. Sublingual buprenorphine has a much longer half-life than parenteral buprenorphine because of sequestration in buccal and sublingual fat. Because transdermal buprenorphine is not commercially available in the US, most clinicians who use the drug often convert from intravenous/subcutaneous infusions to sublingual buprenorphine.

Buprenorphine is a lipophilic, synthetic opioid; these properties, combined with its low molecular weight, make it suitable for transdermal delivery. Average half-life is 37 hours. Its properties of high affinity and slow dissociation combined in a slow-release transdermal form can produce long-term pain relief with fewer side effects, making it suitable for treating persistent chronic pain of long duration in the elderly in whom wide swings of drug concentration are less well tolerated. Transdermal buprenorphine has a half-life of about 30 hours. Sublingual buprenorphine can be given for breakthrough pain. In a long-term study, sublingual buprenorphine was available for use as needed for control of pain (Likar and Sittle 2005).

The transdermal route is especially useful for drugs such as buprenorphine which have limited bioavailability if given orally. Transdermal buprenorphine can provide pain relief for cancer and for chronic noncancer pain (Likar et al 2003; Pavelka et al 2004; Muriel et al 2005). Transdermal buprenorphine formulated in an adhesive polymer matrix (acrylate vinyl acetate) is being widely used clinically for the treatment of moderate to severe cancer pain (Budd and Collett 2003; Sittl et al 2006). The transdermal buprenorphine patch available in matrix form does not allow for dose dumping in the event of damage to the patch and also improves patient compliance, an extremely important factor in elderly patients (Parikh 2007).

The purpose of the transdermal patch is to provide longterm pain relief and to avoid an increase in adverse side effects. The transdermal buprenorphine patch is available in 3 strengths, containing 20,30, or $40 \mathrm{mg}$ of buprenorphine, which can deliver $35,52.5$, or $70 \mu \mathrm{g}$ /hour over 72 hours, respectively. It is generally recommended that patients who have not taken any analgesic or a nonopioid analgesic should start with a $35 \mu \mathrm{g} /$ hour patch. This long-acting form of buprenorphine can be supplemented with the short-acting form of sublingual buprenorphine for breakthrough pain. If the patch is used, the time to minimum therapeutic concentration is 21 hours. After the placement of a transdermal patch additional analgesics should be available as needed for 24-48 hours for treatment of breakthrough pain, because plasma levels of buprenorphine rise slowly after patch application. All opioid-naïve patients, especially the elderly, should be started with the lowest strength patch.

\section{Transdermal buprenorphine for treatment of chronic pain}

Transdermal buprenorphine has been shown to be useful for the treatment of chronic pain associated with cancer as well as noncancer etiologies (Sittl et al 2003). Likar et al (2003) studied the analgesic efficacy and tolerability of transdermal buprenorphine in patients with inadequately controlled chronic pain. They demonstrated that buprenorphine is an effective analgesic for chronic pain. It was shown that transdermal buprenorphine patches are useful for the treatment of moderate to severe cancer pain and also for noncancer pain that is severe and unresponsive to nonopioid analgesics. About one third of study patients with chronic pain had satisfactory analgesia with the administration of buprenorphine. In addition transdermal buprenorphine was found to decrease the need for rescue medications and increases the duration of pain-free sleep. Likar et al (2006) also studied the efficacy and tolerability of long-term treatment with transdermal buprenorphine patients with chronic persistent pain of moderate to severe intensity who had previously received buprenorphine in 3 short-term clinical trials. They studied 134 patients with cancer-related pain and 105 patients with pain of noncancerous origin. The mean duration of participation was 7.5 months and the adherence to therapy was $78.7 \%$. This level of adherence to therapy would be of particular help in the elderly.

Neuropathic pain or chronic persistent pain often presents as burning, lancinating pain caused by injury or chronic 
changes to peripheral or central nerves (McQuay 2002) and has been found to be difficult to treat with conventional analgesics (Foley 2003). There is increasing evidence that opioids can decrease neuropathic pain in peripheral and central areas though the response can be variable (Rowbotham et al 2003). For example morphine, a typical mu opioid agonist, is not very sensitive in treating neuropathic pain (Mao et al 1995). It has been suggested that the ineffectiveness of morphine in controlling neuropathic pain may be due to the downregulation of opioid receptors after nerve injury in the sensory neurons and in the spinal cord (Zhang et al 1998). In contrast, buprenorphine, a stronger opioid, has been shown in rats and humans to be effective in controlling neuropathic pain. It is known that some opioids have an NMDA blocking effect. Ebert et al (1995, pp. 165-8) have shown that buprenorphine is one such opioid that can block NMDA receptors and reduce reflex facilitation and central sensitization (Kouya et al 2002).

Mechanical and cold allodynia and hyperalgesia were alleviated in rats with neuropathic pain behaviors (Kouya et al 2002). Human studies have also shown similar results. Intravenous buprenorphine has been shown to relieve longterm neuropathic pain in patients after thoracotomy (Benedetti et al 1998). In another study, phantom limb pain was relieved by intrathecal buprenorphine after amputation (Omote et al 1995). Buprenorphine given by the transdermal route has also providef adequate pain relief in cases of neuropathic pain, as shown in 3 patients with chronic nonmalignant pain due to musculoskeletal diseases (Balint 2002).

Transdermal buprenorphine has been used for "opioid rotation", which is a method to treat chronic pain refractory to treatment by other opioids for long-term pain relief. In a opioid rotation study (Freye et al 2007), 42 patients (mean age 64.1 years) receiving high-dose morphine (120-240 $\mathrm{mg} /$ day) for chronic pain secondary to neuropathic, musculoskeletal, or malignant etiology were switched to transdermal buprenorphine because of intolerable side effects and insufficient pain relief. The patients were followed for more than 10 weeks. Patients reporting very good pain relief increased from $5 \%$ to $75 \%$ and those reporting improved quality of sleep increased from $14 \%$ to $74 \%$. There was no tolerance to buprenorphine via this route and only $11.9 \%$ of adverse effects were noted, mostly due to local irritation. Chronic pain of cancer and noncancer origin was also reported to be effectively treated in all ages $>70$ years in another study by Griessinger et al (2005), in which a total of 13,179 chronic pain patients were evaluated. In this study $28 \%$ had cancer pain and $72 \%$ had noncancer pain.
No development of tolerance was seen clinically in these patients and the patches were well tolerated. In a randomized controlled study by Pace et al (2007), patients with chronic cancer had better pain control, and improved mental health and vitality with the use of transdermal buprenorphine compared with sustained-release morphine. Transdermal buprenorphine appears to be well tolerated and effective for the treatment of chronic cancer and chronic noncancer pain in the long term.

\section{Advantages in the elderly}

Elderly patients need special consideration with several factors related to drug delivery, including drug delivery itself, drug interactions, and adherence to prescribed regimens. Transdermal drug delivery systems have advantages over other routes of drug administration especially in the elderly (Pepe et al 1988). The transdermal route is suited for the elderly as it provides ease of use for the patient and the caretaker, provides greater adherence to prescribed regimens, and has less risk of toxicity and dose dumping. This is especially important because of the increased need for medications in older age when there is a higher incidence of chronic conditions such as chronic pain, diabetes mellitus, cardiovascular disease, and neurological diseases such as Parkinson's disease and Alzheimer's disease. A transdermal delivery system is also useful when elderly patients are unable to tolerate oral medications or are unwilling to swallow oral medications. Transdermal medications also avoid needle punctures associated with subcutaneous or intravenous routes and are less labor intensive than these routes (Jiang et al 1997). It has been stated that transdermal delivery results in reasonably constant plasma drug concentrations because of its rate controlled delivery.

Transdermal buprenorphine has several advantages over the conventional methods of administration in the elderly: it results in no peak trough effects and a slower increase in serum concentration, resulting in fewer adverse events in serum concentrations compared with sublingual buprenorphine (Bohme 2002; Johnson et al 2005). The use of drugs with an absence of peak trough effects is in accordance with WHO guidelines, which recommend that to decrease adverse side effects, drugs should be used to treat chronic pain that do not induce sudden peaks in serum concentrations. The absorption of transdermal buprenorphine among patients varies less than among patients on transdermal fentanyl (Jensen et al 2007). Transdermal fentanyl absorption has been thought to decrease in the elderly, resulting in many dose adjustments. Since the absorption of transdermal 
buprenorphine is little affected with age, its relative potency will increase in the elderly. It has been suggested that fewer dosage adjustments are needed in patients using transdermal buprenorphine compared with patients using transdermal fentanyl. This was shown in a retrospective analysis on data from 400 medical practices in Germany which showed a significantly greater increase in mean daily dosage in patients treated with transdermal fentanyl compared with patients treated with transdermal buprenorphine (Sittl et al 2006). The painful conditions included cancer, osteoarthritis, low back pain, and osteoporosis.

The reduced need to escalate transdermal buprenorphine relative to transdermal fentanyl is probably related to the antihyperalgesic effect, which prevents or blocks hyperadaptive responses differing from fentanyl (Célèrier et al 2000; Angst et al 2003; Koppert et al 2005). Incidence of chronic pain conditions increases with advancing age. Increased back pain due to osteoporotic and compression fractures are common in the elderly (Gandy and Payne 1986) and transdermal buprenorphine, by virtue of its dosage stability, could be used to treat these conditions safely in the elderly.

The low incidence of adverse events associated with buprenorphine lends an added attraction for use in the elderly who have decreased tolerance to adverse effects. In the elderly the transdermal patch is ideal because it can be easily applied, resulting in greater compliance in this population who tend to be more forgetful. It also has a lower susceptibility to the development of toxicity and opioid abuse because it adheres strongly and is less susceptible to damage (Budd 2003).

\section{Less respiratory depression}

Because of its partial agonist activity and the associated ceiling effect in respiratory depression (Walsh et al 1995), transdermal buprenorphine is useful for treatment in the elderly who are more prone to respiratory depression associated with common respiratory diseases such as chronic bronchitis, emphysema, and chronic obstructive pulmonary disease. Downing et al (1979) studied 10 critically ill patients in whom intravenous buprenorphine $0.2-0.4 \mathrm{mg}$ had no significant effect on base excess values, oxygen saturation, or heart rate. It did, however, increase the arterial carbon dioxide levels and reduce respiratory rate in these patients. A large study in 3,690 cancer patients (44\% of whom were $\geq 70$ years of age) clearly showed the effectiveness and safety of the transdermal buprenorphine patch in the elderly (Griessinger et al 2005).

\section{Long duration of action}

The high affinity to the mu receptor enables buprenorphine to exert its analgesic effect for a long duration, eliminating the need for daily dosing and overcoming problems of nonadherence to treatment regimens which is common in the elderly. This property will also decrease the risk of death by overdose.

\section{Drug interactions in the elderly}

To establish specific safety guidelines, the use of buprenorphine in the elderly with other drugs requires further investigation, especially as many elderly patients are using many different pharmacological agents such as treatment for heart disease and cognitive dysfunction. Common medications of the elderly include beta blockers, statins, and ACE-inhibitors. Buprenorphine has been known to inhibit the cytochrome P 450 system by inhibiting the CYP3A4- and CYP2D6-mediated reactions. It is thought, however, that the clinical concentrations used are unlikely to affect significantly metabolism of drugs that are metabolized by cytochrome P 450 (Umehara et al 2002). It is known than CYP3A4 and UCTiA3 are relatively preserved in the elderly. Drugs that interact with CYP3A4 will not prevent conjugation, which is rate limiting. Buprenorphine interacts with CYP3A4 differently than methadone. It is seem that fluvoxamine, which blocks CYP3A4 and methadone metabolism, does not interfere with buprenorphine clearance (Iribarne et al 1998).

Buprenorphine cannot be dialyzed and very high doses of naloxone are sometimes needed to reverse respiratory depression (Orwin et al 1976; Knape 1986; Gal 1989), because of the high affinity of buprenorphine to the mu receptor as well as the slow dissociation from the receptor. Respiratory depression with the use of buprenorphine, except after the intraspinal route (Chrubasik et al 1994), is very rare (Ventafridda et al 1983). It must be remembered that concomitant exposure to other drugs that can induce or inhibit the enzyme may intensify the action of buprenorphine. Opioids, anesthetic agents, sedatives, hypnotics, monoamine oxidase inhibitors, antidepressants, neuroleptics, and alcohol can intensify the CNS effects of buprenorphine.

\section{Disadvantages in the elderly}

To avoid opioid toxicity symptoms, buprenorphine should be used with caution when it is being used with other drugs such as benzodiazepines. This interaction with benzodiazepines can occur with all opioids and is related to the synergistic 
effect on the CNS causing sedation and respiratory depression. This effect is not due to hepatic metabolism (Ibrahim et al 2000).

Lai and Teo (2006) studied 21 buprenorphine-related deaths in Singapore. In 19 of these deaths buprenorphine and benzodiazepines were being used concurrently, suggesting that their concurrent use could be fatal. All these individuals were under the age of 40 years. Therefore, benzodiazepines should be prescribed with caution in the elderly. It has been reported that intravenous use of buprenorphine combined with benzodiazepines has resulted in some deaths (Reynaud et al 1998). In the event of respiratory depression when transdermal buprenorphine and benzodiazepines are used concurrently, the treatment would include respiratory support with mechanical ventilation if necessary until buprenorphine is completely metabolized.

\section{Buprenorphine and drug dependence}

Buprenorphone is licensed to treat drug dependence. In the large experience published, there are fewer deaths with buprenorphine maintenance therapy than with methadone. If a patient with cancer and opioid dependence or a history of drug abuse had pain, buprenorphine would be a reasonable choice in combination with naloxone.

\section{Use in the presence of cognitive disorders}

In the elderly suffering from cognitive and motor deficits as a result of chronic neurological diseases such as Parkinson's disease and Alzheimer's disease, transdermal buprenorphine will be a useful tool for the administration of drugs when patients are forgetful, or unwilling or unable to swallow oral medications. Cumbersome equipment associated with the use of intravenous of subcutaneous infusions can also be avoided. Rate-controlled delivery through the transdermal route leads to constant plasma concentrations of the drug irrespective of the drug's half-life.

Buprenorphine is metabolized by the liver by glucuronidation and not oxidation, and should be used in caution with elderly patients with hepatic disease such as cirrhosis, though glucuronidation is less affected by liver disease than is oxidation of opioids. In patients with increased temperature, additional monitoring may be required since increased body temperature can increase skin permeability. Conditions where buprenorphine are contraindicated are patients with opioid dependence, myasthenia gravis, respiratory depression, and delirium tremens.

\section{Future research}

In addition to cancer and arthritis, there are several chronic neuropathic pain syndromes that occur more commonly with advancing age. These include post-herpetic neuralgia (Bowsher 1999), diabetes, and strokes. Long-standing diabetes could lead to painful diabetic neuropathy, and strokes can be followed by post-stroke pain. The use of transdermal buprenorphine in these settings will require further evaluation.

\section{Conclusion}

Chronic pain in the elderly is a significant problem and the age-related metabolic, cognitive, and pharmacokinetic changes associated with advanced age make pain control in the elderly a challenge. The transdermal buprenorphine matrix allows for slow release of buprenorphine, and damage does not produce dose dumping, which is an added advantage especially in the elderly (Budd 2003). The high incidence of coexisting diseases such as diabetes, cardiovascular, and neurological diseases in the elderly raises concerns of drug interactions with multiple medications. In addition the elderly need special consideration for adherence to medication regimen and drug delivery (Priano et al 2006). The recently developed transdermal buprenorphine, with its unique pharmacodynamics and special matrix formulation, shows promise for the control of this difficult problem in the elderly. The pharmacodynamics of buprenorphine shows that it has several advantages for use in the elderly. Scientific studies have significantly refuted misconceptions of buprenorphine in the past, such as the inability to produce adequate analgesia because of its partial agonist effect, the ineffectiveness of other opioids to act in the presence of buprenorphine, and the concern that if respiratory depression occurred it could not be reversed with naloxone. Recent scientific studies with buprenorphine have shown no evidence of a ceiling dose of analgesia in humans but only a ceiling effect for respiratory depression (Walsh et al 1995), suggesting a low abuse liability and increased safety. Its mu agonist properties of long duration exist for analgesia, sedation, euphoria, and papillary constriction. Naloxone has been found to reverse any respiratory depression caused by buprenorphine (Gal 1989). The safe and effective use of transdermal buprenorphine in the presence of renal failure makes it an attractive choice for older individuals who have a higher predisposition for the development of renal insufficiency. In addition the low potential for drug to drug interaction along with the beneficial effects on the immune system makes it a suitable opioid for use in the 
elderly (Griessinger et al 2005). It must be remembered, however, that buprenorphine and transdermal buprenorphine could be associated with sedation and the elderly should be monitored carefully for this side effect. In addition buprenorphine should be used with caution with other drugs such as benzodiazepines. More outcome studies are needed on the effectiveness of transdermal buprenorphine for control of chronic pain associated with conditions more commonly seen in the elderly such as diabetic neuropathy, post-stroke pain, and post-herpetic neuralgia.

\section{Disclosures}

Neither author has any conflicts of interest to declare.

\section{References}

Abrahm J. 2000. Advances in pain management for older adult patients. Clin Geriatr Med, 16:269-311.

Angst M, Koppert W, Pahl I, et al. 2003. Short-term infusion of the muopioid agonist remifentanil in humans causes hyperalgesia during withdrawal. Pain, 106:49-57.

Balint G. 2002. Buprenorphine treatment of patients with non-malignant musculoskeletal diseases. Clin Rheumatol, 21(Suppl 1):S17-8.

Balázs E, Ruszwurm A, Székely M, et al. 2008. [Old age and kidneys.]. Orv Hetil, 149:789-94.

Benedetti F, Vighetti S, Amanzio M, et al. 1998. Dose-response relationship of opioids in nociceptive and neuropathic postoperative pain. Pain, 74:205-11.

Bloms-Funke P, Gillen C, Schuettler A, et al. 2000. Agonistic effects of the opioid buprenorphine on the nociceptin/OFQ receptor. Peptides, 21:1141-6.

Bohme K. 2002. Buprenorphine in a transdermal therapeutic system-a new option. Clin Rheumatol, 21(Suppl 1):S13-6.

Book S, Myrick H, Malcolm R, et al. 2007. Buprenorphine for postoperative pain following general surgery in a buprenorphine-maintained patient. Am J Psychiatry, 164:979.

Bowsher D. 1999. The lifetime occurrence of Herpes zoster and prevalence of post-herpetic neuralgia:A retrospective survey in an elderly population. Eur J Pain, 3:335-42.

Brewster D, Humphrey MJ, McLeavy MA. 1981. Biliary excretion, metabolism and enterohepatic circulation of buprenorphine. Xenobiotica, 11:189-96.

Budd K. 2003. Buprenorphine and the transdermal system:the ideal match in pain management. Int J Clin Pract, (Suppl):9-14; discussion 23-4.

Budd K, Collett BJ. 2003. Old dog-new ma.trix. Br J Anaesth, 90:722-4.

Casten RJ, Parmelee PA, Kleban MH, et al. 1995. The relationships among anxiety, depression, and pain in a geriatric institutionalized sample. Pain, 61:271-6.

Chrubasik J, Martin E, Chrubasik S, et al. 1994. Epidural buprenorphine. How safe is "safe". Acta Anaesthesiol Scand, 38:525-6.

Clarot F, Proust B, Vaz E, et al. 2003. Tramadol-benzodiazepines and buprenorphine-benzodiazepines:two potentially fatal cocktails? J Clin Forensic Med, 10:125-6.

Cleeland CS, Gonin R, Hatfield AK, et al. 1994. Pain and its treatment in outpatients with metastatic cancer. N Engl J Med, 330:592-6.

Cone EJ, Gorodetzky CW, Yousefnejad D, et al. 1984. The metabolism and excretion of buprenorphine in humans. Drug Metab Dispos, 12:577-81.

Crook J, Rideout E, Browne G. 1984. The prevalence of pain complaints in a general population. Pain, 18:299-314.

Célèrier E, Rivat C, Jun Y, et al. 2000. Long-lasting hyperalgesia induced by fentanyl in rats:preventive effect of ketamine. Anesthesiology, 92:465-72.
D'elia M, Patenaude J, Hamelin C, et al. 2003. No detrimental effect from chronic exposure to buprenorphine on corticosteroid-binding globulin and corticosensitive immune parameters. Clin Immunol, 109:179-87.

Dahan A, Yassen A, Bijl H, et al. 2005. Comparison of the respiratory effects of intravenous buprenorphine and fentanyl in humans and rats. Br J Anaesth, 94:825-34.

Dahan A, Yassen A, Romberg R, et al. 2006. Buprenorphine induces ceiling in respiratory depression but not in analgesia. Br J Anaesth, 96:627-32.

Davis M, Srivastava, M. 2003. Demographics, assessment and management of pain in the elderly. Drugs Aging, 20:23-57.

Downing JW, Goodwin NM, Hicks J. 1979. The respiratory depressive effects of intravenous buprenorphine in patients in an intensive care unit. S Afr Med J, 55:1023-7.

Ebert B, Andersen S, Krogsgaard-Larsen P. 1995. Ketobemidone, methadone and pethidine are non-competitive N-methyl-D-aspartate NMDA. antagonists in the rat cortex and spinal cord. Neurosci Lett, 187:165-8.

Evans H, Easthope S. 2003. Transdermal buprenorphine. Drugs, 63:1999-010; discussion 2011-2.

Filitz J, Griessinger N, Sittl R, et al. 2006. Effects of intermittent hemodialysis on buprenorphine and norbuprenorphine plasma concentrations in chronic pain patients treated with transdermal buprenorphine. Eur J Pain, 10:743-8.

Foley KM. 2003. Opioids and chronic neuropathic pain. N Engl J Med, 348:1279-81.

Freye E, Anderson-Hillemacher A, Ritzdorf I, et al. 2007. Opioid rotation from high-dose morphine to transdermal buprenorphine Transtec. in chronic pain patients. Pain Pract, 7:123-9.

Gagliese L, Melzack R. 1997. Chronic pain in elderly people. Pain, 70:3-14.

Gal TJ. 1989. Naloxone reversal of buprenorphine-induced respiratory depression. Clin Pharmacol Ther, 45:66-71.

Gandy S, Payne R. 1986. Back pain in the elderly:updated diagnosis and management. Geriatrics, 41:59-62, 67-74.

Gomez-Flores R, Weber R. 2000. Differential effects of buprenorphine and morphine on immune and neuroendocrine functions following acute administration in the rat mesencephalon periaqueductal gray. Immunopharmacology, 48:145-56.

Graziotti PJ, Goucke CR. 1997. The use of oral opioids in patients with chronic non-cancer pain. Management strategies. Med J Aust, $167: 30-4$.

Greenwald M, Johanson C, Moody D, et al. 2003. Effects of buprenorphine maintenance dose on mu-opioid receptor availability, plasma concentrations, and antagonist blockade in heroin-dependent volunteers. Neuropsychopharmacology, 28:2000-9.

Griessinger N, Sittl R, Likar R. 2005. Transdermal buprenorphine in clinical practice - a post-marketing surveillance study in 13,179 patients. Curr Med Res Opin, 21:1147-56.

Grond S, Radbruch L, Lehmann KA. 2000. Clinical pharmacokinetics of transdermal opioids:focus on transdermal fentanyl. Clin Pharmacokinet, 38:59-89.

Hand C, Sear J, Uppington J, et al. 1990. Buprenorphine disposition in patients with renal impairment:single and continuous dosing, with special reference to metabolites. Br J Anaesth, 64:276-82.

Heel RC, Brogden RN, Speight TM, et al. 1979. Buprenorphine:a review of its pharmacological properties and therapeutic efficacy. Drugs, 17:81-110.

Helme RD, Katz B, Gibson S, et al. 1989. Can psychometric tools be used to analyse pain in a geriatric population? Clin Exp Neurol, 26:113-7.

Ibrahim R, Wilson J, Thorsb Y, et al. 2000. Effect of buprenorphine on CYP3A activity in rat and human liver microsomes. Life Sci, 66:1293-8.

Iribarne C, Picart D, Dréano Y, et al. 1998. In vitro interactions between fluoxetine or fluvoxamine and methadone or buprenorphine. Fundam Clin Pharmacol, 12:194-9. 
Jasinski DR, Pevnick JS, Griffith JD. 1978. Human pharmacology and abuse potential of the analgesic buprenorphine:a potential agent for treating narcotic addiction. Arch Gen Psychiatry, 35:501-16.

Jensen M, Foster D, Upton R, et al. 2007. Population pharmacokinetics of buprenorphine following a two-stage intravenous infusion in healthy volunteers. Eur J Clin Pharmacol, 63:1153-9.

Jiang R, Roberts MS, Prankerd RJ, et al. 1997. Percutaneous absorption of sunscreen agents from liquid paraffin:self-association of octyl salicylate and effects on skin flux. J Pharm Sci, 86:791-6.

Johnson RE, Fudala PJ, Payne R. 2005. Buprenorphine:considerations for pain management. J Pain Symptom Manage, 29:297-326.

Kintz P. 2002. A new series of 13 buprenorphine-related deaths. Clin Biochem, 35:513-6.

Kisely S, Tweddle D, Pugh EW. 1992. Dementia presenting with sore eyes. Br J Psychiatry, 161:120-1.

Knape J. 1986. Early respiratory depression resistant to naloxone following epidural buprenorphine. Anesthesiology, 64:382-4.

Koppert W, Ihmsen H, Körber N, et al. 2005. Different profiles of buprenorphine-induced analgesia and antihyperalgesia in a human pain model. Pain, 118:15-22.

Kouya PF, Hao JX, Xu XJ. 2002. Buprenorphine alleviates neuropathic pain-like behaviors in rats after spinal cord and peripheral nerve injury. Eur J Pharmacol, 450:49-53.

Lai SH, Teo CE. 2006. Buprenorphine-associated deaths in Singapore. Ann Acad Med Singapore, 35:508-11.

Likar R, Griessinger N, Sadjak A, et al. 2003. [Transdermal buprenorphine for treatment of chronic tumor and non-tumor pain]. Wien Med Wochenschr, 153:317-22.

Likar R, Kayser H, Sittl R. 2006. Long-term management of chronic pain with transdermal buprenorphine:a multicenter, open-label, followup study in patients from three short-term clinical trials. Clin Ther, 28:943-52.

Likar R, Sittl R. 2005. Transdermal buprenorphine for treating nociceptive and neuropathic pain:four case studies. Anesth Analg, 100:781-5.

Mao J, Price DD, Mayer DJ. 1995. Experimental mononeuropathy reduces the antinociceptive effects of morphine:implications for common intracellular mechanisms involved in morphine tolerance and neuropathic pain. Pain, 61:353-64.

McQuay HJ. 2002. Neuropathic pain:evidence matters. Eur J Pain, 6 (Suppl A):11-8.

Muriel C, Failde I, Mico JA, et al. 2005. Effectiveness and tolerability of the buprenorphine transdermal system in patients with moderate to severe chronic pain:a multicenter, open-label, uncontrolled, prospective, observational clinical study. Clin Ther, 27:451-62.

Negus SS, Mello NK, Linsenmayer DC, et al. 2002. Kappa opioid antagonist effects of the novel kappa antagonist 5'-guanidinonaltrindole GNTI. in an assay of schedule-controlled behavior in rhesus monkeys. Psychopharmacology Berl, 163:412-9.

Ohtani M, Kotaki H, Nishitateno K, et al. 1997. Kinetics of respiratory depression in rats induced by buprenorphine and its metabolite, norbuprenorphine. J Pharmacol Exp Ther, 281:428-33.

Omote K, Ohmori H, Kawamata M, et al. 1995. Intrathecal buprenorphine in the treatment of phantom limb pain. Anesth Analg, 80:1030-2.

Orwin J, Orwin J, Price M. 1976. A double blind comparison of buprenorphine and morphine in conscious subjects following administration by the intramuscular route. Acta Anaesthesiol Belg, 27:171-81.

Pace MC, Passavanti MB, Grella E, et al. 2007. Buprenorphine in long-term control of chronic pain in cancer patients. Front Biosci, 12:1291-9.

Parikh A. 2007. Principles of geriatric pharmacology. J Indian Med Assoc, 105:282-84.

Pavelka K, Le Loet X, Bjorneboe O, et al. 2004. Benefits of transdermal fentanyl in patients with rheumatoid arthritis or with osteoarthritis of the knee or hip:an open-label study to assess pain control. Curr Med Res Opin, 20:1967-77.

Pepe GJ, Waddell BJ, Stahl SJ, et al. 1988. The regulation of transplacental cortisol-cortisone metabolism by estrogen in pregnant baboons. Endocrinology, 122:78-83.
Priano L, Gasco MR, Mauro A. 2006. Transdermal treatment options for neurological disorders:impact on the elderly. Drugs Aging, 23:357-75.

Reynaud M, Tracqui A, Petit G, et al. 1998. Six deaths linked to misuse of buprenorphine-benzodiazepine combinations. Am J Psychiatry, 155:448-9.

Rowbotham MC, Twilling L, Davies PS, et al. 2003. Oral opioid therapy for chronic peripheral and central neuropathic pain. $N$ Engl $J$ Med, 348:1223-32.

Shah F, Halbe A, Panchal I, et al. 2003. Improvement in postoperative pain relief by the addition of midazolam to an intrathecal injection of buprenorphine and bupivacaine. Eur J Anaesthesiol, 20:904-10.

Simonnet G. 2005. Opioids: from analgesia to anti-hyperalgesia? Pain, 118:8-9.

Simonnet G, Rivat C. 2003. Opioid-induced hyperalgesia: abnormal or normal pain? Neuroreport, 14:1-7.

Simpson KH. 2002. Individual choice of opioids and formulations:strategies to achieve the optimum for the patient. Clin Rheumatol, 21 (Suppl 1): S5-8.

Sittl R. 2006. Transdermal buprenorphine in cancer pain and palliative care. Palliat Med, 20 (Suppl 1):s25-30.

Sittl R, Griessinger N, Likar R. 2003. Analgesic efficacy and tolerability of transdermal buprenorphine in patients with inadequately controlled chronic pain related to cancer and other disorders: a multicenter, randomized, double-blind, placebo-controlled trial. Clin Ther, 25:150-68.

Sittl R, Nuijten M, Nautrup B. 2005. Changes in the prescribed daily doses of transdermal fentanyl and transdermal buprenorphine during treatment of patients with cancer and noncancer pain in Germany: results of a retrospective cohort study. Clin Ther, 27:1022-31.

Sittl R, Nuijten M, Nautrup BP. 2006. Patterns of dosage changes with transdermal buprenorphine and transdermal fentanyl for the treatment of noncancer and cancer pain: a retrospective data analysis in Germany. Clin Ther, 28:1144-54.

Sorge J Sittl R. 2004. Transdermal buprenorphine in the treatment of chronic pain: results of a phase III, multicenter, randomized, double-blind, placebo-controlled study. Clin Ther, 26:1808-20.

Summerfield R, Allen M, Moore R, et al. 1985. Buprenorphine in end stage renal failure. Anaesthesia, 40:914.

Thompson J, Bower S, Liddle A, et al. 1998. Perioperative pharmacokinetics of transdermal fentanyl in elderly and young adult patients. $\mathrm{Br} J$ Anaesth, 81:152-4.

Umehara K, Shimokawa Y, Miyamoto G. 2002. Inhibition of human drug metabolizing cytochrome P450 by buprenorphine. Biol Pharm Bull, 25:682-5.

Vadivelu N, Hines RL. 2007. Buprenorphine: a unique opioid with broad clinical applications. J Opioid Manag, 3:49-58.

Ventafridda V, De Conno F, Guarise G, et al. 1983. Chronic analgesic study on buprenorphine action in cancer pain. Comparison with pentazocine. Arzneimittelforschung, 33:587-90.

Walsh SL, Preston KL, Bigelow GE, et al. 1995. Acute administration of buprenorphine in humans:partial agonist and blockade effects. J Pharmacol Exp Ther, 274:361-72.

Walsh SL, Preston KL, Stitzer ML, et al. 1994. Clinical pharmacology of buprenorphine:ceiling effects at high doses. Clin Pharmacol Ther, 55:569-80.

Woo J, Ho SC, Lau J, et al. 1994. Musculoskeletal complaints and associated consequences in elderly Chinese aged 70 years and over. J Rheumatol, 21:1927-31.

Yassen A, Olofsen E, Dahan A, et al. 2005. Pharmacokinetic-pharmacodynamic modeling of the antinociceptive effect of buprenorphine and fentanyl in rats: role of receptor equilibration kinetics. $J$ Pharmacol Exp Ther, 313:1136-49.

Zaki PA, Keith DE, Jr, Brine GA, et al. 2000. Ligand-induced changes in surface mu-opioid receptor number:relationship to G protein activation? J Pharmacol Exp Ther, 292:1127-34.

Zhang Y, Du LN, Wu GC, et al. 1998. Modulation of intrathecal morphineinduced immunosuppression by microinjection of naloxone into periaqueductal gray. Zhongguo Yao Li Xue Bao, 19:519-22. 